\title{
The Relationship between Future Self-Continuity and Mobile Phone Dependence of College Students: Mediating Role of Self-Control
}

\author{
Guifang $\mathrm{Fu}^{1}$, Siman $\mathrm{Li}^{1}$, Jia Guo ${ }^{2}$ \\ ${ }^{1}$ Department of Applied Psychology, School of Public Administration, Guangdong University of Foreign Studies, Canton, \\ China \\ ${ }^{2}$ Students' Affairs Division, Guangdong University of Foreign Studies, Canton, China \\ Correspondence: Guo Jia, Students' Affairs Division, Guangdong University of Foreign Studies, Canton, China
}

Received: February 25, 2020

Accepted: March 23, $2020 \quad$ Available online: March 26, 2020

doi:10.11114/ijsss.v8i3.4788

URL: https://doi.org/10.11114/ijsss.v8i3.4788

\begin{abstract}
The problem of mobile phone dependence is becoming more and more serious. Therefore, it is very important to explore the causes of mobile phone dependence and its psychological mechanism. One of the important characteristics of mobile phone dependence is the loss of control, which shows that self-control is an important factor affecting mobile phone dependence. Self-continuity is closely related to cell phone dependence. Mobile phone addicts usually use mobile phones to temporarily relieve the pressure and negative emotions, but it will lead to more pressure and negative emotions in the future. In order to examine the situation of college students' future self-continuity, self-control and mobile phone addiction as well as their relationships, especially mediating effect of self-control, a total of 482 college students were assessed with Future Self-Continuity Scale (FSC), Self-Control Scale (SCS), and Mobile Phone Dependency Index (MPAI). The results showed that: (1) The future self-continuity, self-control and mobile phone dependence of the college students in this study were all at a medium level, and there was no significant difference in demographic variables (such as gender, grade, etc.); (2) Both future self-continuity and self-control were negatively correlated with mobile phone dependence; (3) There was a significant positive correlation between future self-continuity and self-control; (4) Self-control played a partial mediating role between future self-continuity and mobile phone dependence. Therefore, improving self-continuity and self-control can be an effective way to intervene mobile phone dependence. In addition research implication, limitations and future directions were discussed.
\end{abstract}

Keywords: future self-continuity, mobile phone dependence, self-control, college students

\section{Introduction}

With the rapid development of the Internet and the popularity of mobile phones, mobile phones have become an indispensable tool for people. They can bring convenience and entertainment to people; however, excessive use of mobile phones will also bring many negative effects on people's lives. Mobile phone dependence, also known as "cell phone addiction" and "problematic use of cell phones," refers to the excessive use of cell phones, which has negative effects on both psychology and physiology (Xiong, Zhou, Chen, et al., 2012). The American Psychological Association (APA) pointed out that excessive use of mobile phones may bring people anxiety and psychological stress, and the daily use of mobile phones by contemporary youth was up to 15-18 hours. Previous studies found that cell phone dependence can lead to problems such as face-to-face interpersonal alienation, low life satisfaction, sleep disturbance, attention deficit and memory loss (Han, Geng, Jou, Gao, Yang, 2017). Among college students, the problem of mobile phones dependence is becoming more and more prominent. It has an impact on the normal study and life of college students in classroom concentration, academic performance, mental health and other aspects (Liu, Zhou, Niu, Fan, 2017).

Therefore the research on the psychological mechanism of college students' cell phone dependence has been an important topic in the field of psychological development. This study will start from a new psychological perspective to study the impact of college students' future self-continuity on mobile phone dependence.

Parfit (1971) thought that the self could be divided into various components in different dimensions. And according to time dimension self is composed of past self, present self and future self. Chandler (1994) first put forward the concept of self-continuity, that is, individuals regard past self, present self and future self as a continuous whole. Future self-continuity refers to the degree to which an individual associates the present self with the future self (Hershfield, 
Garton, Ballard, Samanez-larkin, \& Knutson, 2009a; Hershfield, Wimmer, \& Knutson, 2009b), the higher the individual's future self-continuity, the closer the connection between them. According to the future self-continuity model of Hershfield (2011), future self-continuity is jointly influenced by three dimensions. They are similarity, vividness and positivity. Similarity refers to the degree of similarity between present self and future self. Vividness refers to the vivid picture that comes to mind when an individual imagines himself at a certain future time. Positivity refers to how positive your future self-image is when you imagine yourself at a future point in time. Future self-continuity seriously affects the present self. Firstly, the three dimensions positively affect the future self-continuity, individual of high future self-continuity believes that the present self have a higher similarity with the future self, and can vividly imagine the self-image at a certain point in the future and have a more positive evaluation of it (Liu, Yang, Wang, Chen, Cai, 2018). Secondly, individual with better self continuity have less self procrastination (Blouin-Hudon \& Pychyl, 2015). Future self-continuity can be measured by scale and experimental manipulation. The future self-continuity scale prepared by Hershfield et al. (2009) is usually used, which regards future self-continuity as a trait or individual difference. The experimental manipulation is to arouse the future self-continuity of the subjects through reading, writing and other activities such as having the subjects write a letter to themselves ten years from now (Hershfield, Cohen, \& Thompson, 2012), or reading stories of people who have experienced fewer changes in their lives (Bartels \& Rips, 2010).

Mobile phone dependence is mainly reflected in individuals' strong and continuous sense of dependence and demand for mobile phones (Xu, Wu, Lan, 2008). Previously, scientists have proved that mobile phone dependence was related to personality traits. For example, shyness could significantly predict the level of mobile phone dependence (Han et al., 2017). The lower the level of self-esteem and interpersonal adaptability, the higher the level of mobile phone addiction tendency (Zhang, Lu, Song, Huang, Ma, Zhang, Yang, Jiang, 2018). Abnormal use of mobile phones may also be related to time discounts in inter-temporal decisions. Past researches have found that people's attempts to ease the current negative emotions or escape of the encountered problems and pressures usually cause mobile phone dependence (Kim, Seo, \& David, 2015). As a matter of fact, mobile phone temporarily relieve stress and negative emotions, it might leads more of those in the future (Liu, zhou et al., 2017).

The study of Hershfield (2011) found that future self-continuity would affect individuals' inter-temporal decision-making and was mainly reflected in time discount-individuals pay more attention to their current profits and losses than those in the future (Hershfield et al., 2009; Ablatijiang, Liu, Zhu, Zheng, Liang, Rao, Wu, Li, 2015). Individuals with high future self-continuity believe that the present self is closely related to the future self, so they will attach more importance to the future self's profits and losses, thereby reducing the time discount in inter-temporal decision-making.

So assumption can be raised that college students with high future self-continuity, in comparison with the current pleasure brought by mobile phone, would pay more attention to negative effects of mobile phone overuse and profits and losses in the future, which means their mobile phone dependence level are lower.

Self-control refers to the ability of individuals to restrain their own desires and change the inherent mode of behavior and thinking in order to match their personal value with social expectations (Tan, Guo, 2008). Self-control is one of the subsystems of the self-regulation system. Individuals with good self-control ability can comprehensively and objectively analyze themselves and constantly improve themselves to integrate social value into their self-value, thereby contributing themselves to the society. Self-control is the embodiment of self-regulation at the behavior level and is the last step to achieve a good self-regulation (Peng, 2012).

Adelman et al. (2016) found that self-control played an intermediary role in the influence of future self-continuity on academic achievement of college students' (Adelman, Herrmann, Bodford, Barbour, Graudejus, Okun, \& Kwan, 2016). College students with a high future self-continuity believe that their current behaviors will have a greater impact on their future selves, and pay more attention to their future goals rather than short-term needs, thus triggers individuals to take control of their current behaviors, which helps college students focus on their study and achieve higher academic achievements.

Self-control is also an important predictor of individual's problematic behaviors and addiction. Individuals with strong self-control ability can rationally control their behaviors and reduce their needs to match their personal values with social expectations. Studies have found that self-control has a significant impact on Internet addiction and online game addiction (Han et al., 2017). In addition Internet addiction and procrastination were negatively correlated with core self-evaluation and self-control (Geng ect. 2018). College students with weak self-control are more likely to indulge in their cell phones, which is one of the most convenient tools for people to gain entertainment, relaxation and social behavior in the Internet. The research of Lee and Park (2014) also proves that self-control Self-continuity will affect self-planning and self-control. Out of control is an important feature of mobile phone dependence, which shows that self-continuity is closely related to mobile phone dependence. Therefore, this study aims to explore how self continuity and self-control affect mobile phone dependence. The theory meanings of this research lies in the extension of the new topic of future self-continuity and 
mobile phone addiction and the reconfirmation of the existing research conclusions. The practical value lies in providing empirical evidence and enlightenment for modern college students to reduce their dependence on mobile phones. In general, the following hypotheses are proposed in this study:

Hypothesis 1: future self-continuity is negatively correlated with mobile phone dependence.

Hypothesis 2: future self-continuity is positively correlated with self-control, while cell phone dependence is negatively correlated with self-control.

Hypothesis 3: self-control mediates the future self-continuity and cell phone dependence.

\section{Methods}

\subsection{Participant}

A total of 492 questionnaires were collected, including 482 valid questionnaires (194 males and 288 females). The basic information of subjects was shown in table 1 .

Table 1. Summary of basic information of subjects 1

\begin{tabular}{cccc}
\hline & Attribute & Population & Percentage (\%) \\
\hline Gender & Male & 194 & 40.2 \\
& Female & 288 & 59.8 \\
Grade & A freshman & 120 & 24.9 \\
& A sophomore & 81 & 16.8 \\
& Junior & 96 & 19.9 \\
& Senior & 185 & 38.4 \\
Registered residence & Cities and towns & 288 & 59.8 \\
& Rural & 194 & 40.2 \\
Only child or not & Is & 172 & 35.7 \\
& No & 310 & 64.3 \\
\hline
\end{tabular}

\subsection{Instruments}

The questionnaire used in this study was composed of three parts, including Mobile Phone Addiction Index (MPAI), Future Self-Continuity Scale (FSC) and Self-Control Scale (SCS). The specific situation of each Scale is as follows.

\subsubsection{Mobile Phone Addiction Index}

This scale was compiled by Lueng (2008) with a total of 17 items, including four factors, including uncontrollable, withdrawal, avoidance and inefficiency. The scale uses the five-point scoring method, the higher the score, the higher the degree of mobile phone dependence. In previous studies on college students' mobile phone dependence, the scale shows good reliability and validity (Liang, 2008; Liu, Zhou et al., 2017), Cronbach alpha coefficient was 0. 87.

\subsubsection{Future Self-Continuity Scale}

The Future Self-Continuity Scale was prepared by Hershfield et al. (2009) and revised by Liu, Yang et al. (2018). The scale contains seven sets of graphs; each set of them consists of two circles. The two circles represent the "present self" and "future self" respectively, and the overlapping parts of the two circles represent the extents of connection and similarity between the "present self" and the "future self". The overlaps gradually increase from figure (1) to figure (7). The more overlaps there are, the closer the connection between "present self" and "future self" and the more similar they are. Participants are required to choose a set of graphs that are most suitable for their own situation. Studies have shown that the reliability and validity of the scale is good (Hershfield et al., 2012), and the Cronbach alpha coefficient was 0. 66.

\subsubsection{Self-Control Scale}

The Self-Control Scale for college students revised by Tan and Guo (2008) was adopted. There were 19 items in total, including five factors: impulse control, healthy habits, resisting temptation, job focus and temperance entertainment. The scale adopts the five-point method, in which 15 questions must be scored in reverse. The higher the score is, the stronger the self-control ability of the subject is. The scale has a good reliability and validity in the measurement of college students' self-control in the past (Zhang et al., 2018), and the Cronbach alpha coefficient was 0. 845.

\subsection{Research Process}

A cross-sectional survey was conducted from January to April 2019. A convenience cluster sampling method was employed to produce a sample of college students. All participants were recruited from Guangdong province. Everybody involved in this study will be told to participant voluntarily and could withdraw at any time. The participants answered a questionnaire through Wenjuanxing on the Internet. It took the respondents approximately 10 min to complete the 
anonymous questionnaire. A small red bag was given to make up for the time spent on the survey.

\subsection{Data Processing}

SPSS 18. 0 was used for data statistics and analysis. The statistical methods are descriptive statistics, correlation analysis, hypothesis testing and regression analysis.

\section{Results}

\subsection{Status Quo of College Students' Mobile Phone Use and basic Information of Various Variables}

According to the descriptive statistics, $50 \%$ of college students use mobile phones for more than 5 hours on average every day, $22.4 \%$ of college students choose to buy mobile phones with a price of more than $¥ 5,000$, and they use social software or websites such as WeChat, weibo, etc., online payment, information accessing and other functions most.

Table 2. Mean and standard deviation of each variable and its sub-dimensions

\begin{tabular}{cccc}
\hline & $N$ & $M$ & $S D$ \\
\hline Phone addiction - uncontrollable & 482 & 2.35 & 0.66 \\
Phone addiction - withdrawal & 482 & 2.61 & 1.03 \\
Phone addiction - avoidance & 482 & 3.02 & 1.02 \\
Phone addiction - inefficiency & 482 & 3.20 & 0.92 \\
Total MPAI & 482 & 2.68 & 0.66 \\
Impulse control & 482 & 3.22 & 0.76 \\
Healthy habits & 482 & 2.81 & 0.75 \\
Resisting temptation & 482 & 2.88 & 0.58 \\
Job focus & 482 & 2.86 & 0.62 \\
Temperance entertainment & 482 & 3.26 & 0.71 \\
Total SCS & 482 & 3.03 & 0.54 \\
Future self-continuity & 482 & 3.89 & 1.43 \\
\hline
\end{tabular}

As shown in table 2, subjects' mobile phone dependence was generally at the medium level $(M=2.68)$. The scale had the lowest score of 1 and the highest score of 5, and "uncontrollable" and "inefficiency" have the highest score. That is to say, college students' mobile phone dependence is mainly reflected in their addiction to mobile phones in order to escape from negative emotions or realistic pressure, which leads to reduced study or work efficiency. Participants' future self-continuity was generally at the medium level $(M=3.89)$, and the lowest to highest score is 1 to 7 . Subjects' self-control was generally at the medium level $(M=3.03)$, and the lowest to highest score is 1 to 5 , with stronger self-control ability in impulse control $(M=3.22)$ and temperance entertainment $(M=3.26)$.

\subsection{Difference Tests of the Three Variables on Demographic Variables}

Independent Sample T Test and one-factor analysis of variance were respectively used to examine whether there are demographic differences in mobile phone dependence $\left(t_{1} 、 F_{1}\right)$, future self-continuity $\left(t_{2} 、 F_{2}\right)$ and self-control $\left(t_{3} 、 F_{3}\right)$. The results showed that there were no significant differences in these three variables by gender $\left(t_{1}=1.14 ; t_{2}=0.11 ; t_{3}=\right.$ $0.89)$, registered residence $\left(t_{1}=0.13 ; t_{2}=1.00 ; t_{3}=0.96\right)$, only child or not $\left(t_{1}=0.29 ; t_{2}=0.94 ; t_{3}=1.66\right)$, and grade $\left(F_{1}=\right.$ $0.35 ; F_{2}=2.08 ; F_{3}=1.66$ ). Therefore, the effect of these demographic variables on results can be excluded in subsequent statistical analysis.

\subsection{Correlation Analysis among College Students' Mobile Phone Dependence, Future Self-Continuity and Self-Control}

\subsubsection{Correlation among variables}

Table 3. Correlation of cell phone dependence, future self-continuity and self-control

\begin{tabular}{|c|c|c|c|}
\hline & Future self continuity & Self control & Mobile phone rely on \\
\hline Future self-continuity & 1.00 & & \\
\hline Self-control & $0.14 * *$ & 1.00 & \\
\hline Mobile phone dependence & $-0.10 *$ & $-0.58 * *$ & 1.00 \\
\hline
\end{tabular}

$* P<0.05 ; * * P<0.01$.

Pearson correlation was adopted to examine whether there was a significant correlation among college students' mobile phone dependence, future self-continuity and self-control, as shown in table 3. Mobile phone dependence was negatively 
correlated with future self-continuity, therefore hypothesis 1 proved. Cell phone dependence was negatively correlated with self-control, and future self-continuity was positively correlated with self-control, thereby hypothesis 2 was confirmed.

\subsection{Mediation Effect}

According to the previous correlation analysis, college students' future self-continuity could significantly predicts mobile phone dependence, which satisfies the premise condition of intermediary test, that is, independent variables significantly affect dependent variables (Wen, Zhang, Hou, Liu, 2004). To further test the relationship among mobile phone dependence, future self-continuity and self- control, and to explore whether self-control play an intermediary effect between future self-continuity and mobile phone dependence, the sequential test method of mediating effect (Wen et al., 2004) was adopted, and three linear regression equations were established for data analysis. The results were as shown in table 4.

Table 4. Mediating effect test results

\begin{tabular}{|c|c|c|c|c|c|c|}
\hline & The independent variables & $\begin{array}{c}\text { The dependent } \\
\text { variable }\end{array}$ & $R^{2}$ & $F$ & $p$ & $\begin{array}{c}\text { Non standardized } \\
\text { coefficient B }\end{array}$ \\
\hline Equation 1 & Future self continuity & $\begin{array}{l}\text { Mobile phone } \\
\text { rely on }\end{array}$ & 0.009 & 4.579 & $0.033^{*}$ & -0.045 \\
\hline Equation 2 & Future self continuity & Self control & 0.020 & 10.025 & $0.002 * *$ & -0.054 \\
\hline Equation 3 & $\begin{array}{c}\text { Future self continuity } \\
\text { Self control }\end{array}$ & $\begin{array}{l}\text { Mobile phone } \\
\text { rely on }\end{array}$ & 0.336 & 120.997 & $\begin{array}{l}0.697 \\
0.000 * * *\end{array}$ & $\begin{array}{l}-0.007 \\
-0.708 \\
\end{array}$ \\
\hline
\end{tabular}

$* P<0.05 ; * * P<0.01 ; * * * P<0.001$

According to regression equation 1 and 2, there was a significant negative correlation between future self-continuity and mobile phone dependence and a significant positive correlation between future self-continuity and self-control. On the basis of equation 3, self-control played a partial mediating role between future self-continuity and mobile phone dependence, which means the influence of future self-continuity on cellphone dependence is mediated by self-control. Therefore hypothesis 3 proved.

\section{Discussions}

\subsection{Demographic Differences among the Three Variables}

This study found that college students' mobile phone dependence was at the middle level, and demographic variables such as gender, grade and registered residence did not affect the results. However, existing studies found that age is an important factor affecting future self-continuity (Chiu, 2015; Jiang \& Zhao, 2016). With the increase of age, the consistency between present self and future self-perceived by individuals increases (Liu et al., 2018). Women are more likely to use mobile phones to establish and maintain social interaction (Bianchi \& Phillips, 2005), and women spend more time and feelings in shopping and interpersonal communication (Rees \& Noyes, 2007). In the study of mobile phone dependence on sleep quality of adolescents, Liu et al. (2017) have controlled gender and age differences. We assume that what we have concluded in this study - there was no age difference in each variables - may be because of the small age difference for the subjects are all college students. Therefore, future self-continuity was not significantly different among grade groups, and it needs to be improved in future studies.

\subsection{Self-Control and Mobile Phone Dependence}

In terms of self-control and mobile phone dependence, the results showed that there is a significant negative correlation between the them, the stronger the self-control ability of college students, the lower the degree of mobile phone dependence they might have, which is the same as the previous research results. It found that self-control is an important index to predict individual's addictive behavior. Facing with the temptation of entertainment and information from the Internet, individuals with strong self-control ability can rationally control the time they spend on mobile phones and reduce their own needs to match their personal value with social expectations (Zhang et al., 2018). In addition, the use of mobile phones can significantly reduce individual self-control (Chen, 2016). When people using their mobile phones, they need to consume psychological resources for information acquisition and processing, self-expression, interpersonal communication and other aspects, so the reduce of psychological resources eventually resulting in the failure of self-control (Baumeister, Vohs, \& Tice, 2007).

\subsection{Future Self-Continuity and Self-Control}

The results showed that there was a significant positive correlation between future self-continuity and self-control, which is consistent with previous research results. The higher the future self-continuity of individuals is, the closer they think the 
present self is connected with the future self, and they would pay more attention to the influence of their current behaviors on the future consequences. Future self-continuity is also an important factor affecting inter-temporal decision-making, which is mainly reflected in time discount. Individuals with high future self-continuity would like to wait for higher remuneration (Hershfield, 2011). Future self-continuity will also affect saving and consumption behaviors (Bartels, \& Urminsky, 2015) and the occurrence of bad social behaviors (Liu et al., 2018). While guiding individuals to focus on future goals and consequences can mediate the effect of future self-continuity on self-control, individuals with high future self-continuity will reduce their short-term needs and pay more attention to future goals (Adelman et al., 2016). Therefore, by guiding college students to carry out self-regulation for long-term goals, it is helpful for students to adopt behaviors or decisions more conducive to their future self.

\subsection{Mediating Effect of Self-Control}

The results showed that self-control played a mediating role between future self-continuity and mobile phone dependence. College students with higher future self-continuity will have stronger self-control and lower dependence on mobile phones. They'd like to pay more attention to the effect of their current behaviors on future consequences. In addition selfregulation can be improved through practice (Baumeister, etc. 2010). After comparing the weight of academic achievement and mobile entertainment, they can rationally control the use of mobile phones and focus on the current academic missions or something benefit to self-growth in the future. Qing-Qi, Dong-Jing etc. also found the level of self-control mediates between perceived stress and cell phone addiction (2018).

Similar to this study, future self-continuity will also affect students' academic achievement through self-control (Adelman et al., 2016), and consideration of future results is also significantly positively correlated with students' academic performance. Future self-continuity also improves individuals' expectation of future goals, attaches importance to behaviors that would be helpful to achieve future goals, and promotes individuals to control themselves more effectively. The vividness of future self regulates the change of future self-continuity, while the emotional transference of future self regulates the relationship between future self-vividness and future self-continuity. Finally, only empathy perspective is an important moderator of delay time (Blouin-Hudon \& Pychyl, 2016).

\subsection{Research Implications, Limitations and Future Directions}

Assisting people in using their devices properly and easing their addiction to them is one of the top 10 psychological development themes, according to a report of development trend of psychology published by APA in 2019. An innovation of this study is the first to study the effect of self-continuity on cell phone dependence. The results of the study not only answer the issue how future self-continuity affect on the mobile phone dependence through self-control, also provide evidences of the relationship among future self-continuity, self-control and mobile phone dependence.

The research results also provide college students advices on reducing mobile phone dependence-students can practice self-control effectively by focusing on the future goals, rather than the current mobile phone demand; students can also increase the consistency of the present self and the future self and shape a more positive future self image, which helps to improve the future self-continuity and self-control ability, thereby reducing mobile phone dependence.

The limitations of this study and suggests directions for future researchers are as follows: (1) Compared with the scale measurement, the manipulation experiment can make participants feel the self-continuity in the time dimension, effectively arouse the their imagination of the future self, and obtain more effective data. However, due to limited resources, only questionnaire survey was used in this study, which should be improved in future studies. (2) Since the participants are only limited to college students, the external validity of the research results needs to be verified. Future research should collect data from multiple channels to make it more representative. (3) Future research should take "considering future results" as a variable to further test whether it will play a mediating or moderating role between future self continuity and mobile phone dependence.

\section{Conclusions}

This study explored the influence of college students' future self-continuity on mobile phone dependence and the mediating effect of self-control. The results showed that: (1) the future self-continuity, self-control and mobile phone dependence of college students were all in the medium level, and there was no significant difference in demographic variables (such as gender, grade, etc.). (2) Future self-continuity and self-control were negatively correlated with mobile phone dependence; (3) Future self-continuity was positively correlated with self-control; (4) future self-continuity and self-control were negatively correlated with mobile phone dependence, while future self-continuity influenced mobile phone dependence through the mediating role of self-control.

\section{Acknowledgements}

This work was supported by The National Social Science Fund of China [grant number 16BSH094]. 


\section{References}

Ablatijiang, L. Y., Zhu, X., Zheng, R., Liang, Z., Rao, L., Wu, B., \& Li, S. (2015). Self-influence on intertemporal decision-making -- based on individual-collectivism cultural perspective. Advances in Psychological Science, 23(11), 1981-1990. https://doi.org/10.3724/SP.J.1042.2015.01981

Adelman, R. M., Herrmann, D. D., Bodford, J. E., Barbour, J. E., Graudejus, O., Okun, M. A., \& Kwan, V. S. (2017). Feeling closer to the future self and doing better: Temporal Psychological Mechanisms Underlying Academic Performance. Journal of Personality, 85(3), 398-408. https://doi.org/10.1111/jopy.12248

Bartels, D. M., \& Rips, 1. J. (2010). Psychological connectedness and intertemporal choice. Journal of Experimental Psychology: General, 139(1), 49-69. https://doi.org/10.1037/a0018062

Bartels, D. M., \& Urminsky, O. (2015). To know and care: How awareness and valuation of the future jointly shape consumer spending. Journal of consumer Research, 41(6), 1469-1485. https://doi.org/10.1086/680670

Baumeister, R. F., Gailliot, M., Dewall, C. N., \& Oaten, M. (2010). Self-regulation and personality: how interventions increase regulatory success, and how depletion moderates the effects of traits on behavior. Journal of Personality, 74(6), 1773-1802. https://doi.org/10.1111/j.1467-6494.2006.00428.x

Baumeister, R. F., Vohs, K. D., \& Tice, D. M. (2007). The strength model of self-control. Current Directions in Psychological Science, 16, 351-355. https://doi.org/10.1111/j.1467-8721.2007.00534.x

Bianchi, A., \& Phillips, J. G. (2005). Psychological predictors of problem mobile phone use. CyberPsychology \& Behavior, 8(1), 39-51. https://doi.org/10.1089/cpb.2005.8.39

Blouin-Hudon, E. M. C., \& Pychyl, T. A. (2015). Experiencing the temporally extended self: initial support for the role of affective states, vivid mental imagery, and future self-continuity in the prediction of academic procrastination. Personality and Individual Differences, 86, 50-56. https://doi.org/10.1016/j.paid.2015.06.003

Blouin-Hudon, E. M. C., \& Pychyl, T. A. (2016). A mental imagery intervention to increase future self-continuity and reduce procrastination. Applied Psychology. https://doi.org/10.1111/apps.12088

Chandler, M. (1994). Self - continuity in suicidal and non suicidal adolescents. New Directions for Child and Adolescent Development, 64, 55-70. https://doi.org/10.1002/cd.23219946406

Chen, W. (2016). The influence of mobile phone use on teenagers' self-control -- based on the interpretive level theory. Wuhan: Central China normal university, PhD Dissertation.

Chiu, S., Hong, F. Y., \& Chiu, S. L. (2015). An analysis on the correlation and gender difference between college students' internet addiction and mobile phone addiction in taiwan. Isrn Addiction, 2013, 360-607. https://doi.org/10.1155/2013/360607

Geng, J., Han, L., Gao, F., Jou, M., \& Huang, C. C. (2018). Internet addiction and procrastination among chinese young adults: a moderated mediation model. Computers in Human Behavior, 84, 320-333. https://doi.org/10.1016/j.chb.2018.03.013

Hershfield, H. E. (2011). Future self - continuity: How conceptions of the Future self transform intertemporal choice. Annals of the New York Academy of Sciences, 1235(1), 30-43. https://doi.org/10.1111/j.1749-6632.2011.06201.x

Hershfield, H. E., Cohen, T. R., \& Thompson, L. (2012). Short horizons and tempting situations: Lack of continuity to our future selves leads to unethical decision making and behavior. Orgnizational Behavior and Human Decision Process, 117(2), 298-310. https://doi.org/10.1016/j.obhdp.2011.11.002

Hershfield, H. E., Garton, M. T., Ballard, K., Samanez-larkin, G. R., \& Knutson, B. (2009). Don't stop thinking about tomorrow: Individual differences in future self-continuity account for saving. Udgment and Decision Making, 4(4), 280-286

Jiang, Z., \& Zhao, X. (2016). Self-control and problematic mobile phone use in Chinese college students: the mediating role of mobile phone use patterns. BMC Psychiatry, 16, 416. https://doi.org/10.1186/s12888-016-1131-z

Kim, E. J., Namkoong, K., Ku, T., \& Kim, S. J. (1971). Philosophical Review, 80(1), 3-27.

Lee, Y. J., \& Park, J. H. (2014). The effect of use withdrawal, self-control and social withdrawal on smartphone addiction. Journal of Digital Convergence, 12(8), 459-465. https://doi.org/10.14400/JDC.2014.12.8.459

Li, L., Mei, S., Niu, Z., \& Song, Y. (2016). The relationship between loneliness and sleep quality in college students: mediating effect and gender regulating effect of smartphone addiction. Chinese Journal of Clinical Psychology, 24, 345-348, 320 . 
Liu, Q., Zhang, D., Yang, X., Zhang, C., Fan, C., \& Zhou, Z. (2018). Perceived stress and mobile phone addiction in Chinese adolescents: a moderated mediation model. Computers in Human Behavior, 87, $247-253$. https://doi.org/10.1016/j.chb.2018.06.006

Liu, Q., Zhou, Z., Niu, G., \& Fan, C. (2017). Cell phone addiction and sleep quality in adolescents: analysis of mediating and regulating effects. Acta psychologica sinica, 49(12), 1524-1536. https://doi.org/10.3724/SP.J.1041.2017.01524

Liu, Y., Yang, Z., Wang, Y., Chen, J., \& Cai, H. (2008). Future self-continuity and its impact on individual psychology and behavior. Progress in psychological science, 26(12), 2161-2169. https://doi.org/10.3724/SP.J.1042.2018.02161

Peng, D. (2012). General psychology. Beijing: Beijing Normal Law Press, 498-499.

Rees, H., \& Noyes, J. M. (2007). Mobile telephones, computers, and the internet: sex differences in adolescents use and attitudes. Cyber Psychology \& Behavior, 10(3), 482-484. https://doi.org/10.1089/cpb.2006.9927

Tan, S., \& Guo, Y. (2008). Revision of self-control scale for college students. Chinese Journal of Clinical Psychology, $16(5), 468-470$.

Wen, Z., Zhang, L., Hou, J., \& Liu, H. (2004). Mediating effect test procedure and it application. Acta psychologica sinica, 36(5), 614-620.

Xiong, J., Zhou, Z., \& Chen, W. (2012). Preparation of mobile phone addiction tendency scale for college students. Chinese Journal of Mental Health, 26(3), 222-225. https://doi.org/10.1037/t74211-000

$\mathrm{Xu}, \mathrm{H}$. , Wu, X., \& Lan, Y. (2008). Preparation of mobile phone dependence scale for college students. Chinese journal of clinical psychology, 16(1), 26-27.

Zhang, Y., Lu, G., Song, X., Huang, H., Ma, X., Zhang, Y., Yang, X., \& Jiang, H. (2018). Mediating effect of self-control and interpersonal adaptability on self-esteem and mobile phone addiction in college students. Chinese Journal of Mental Health, 32(5), 420-424.

\section{Copyrights}

Copyright for this article is retained by the author(s), with first publication rights granted to the journal.

This is an open-access article distributed under the terms and conditions of the Creative Commons Attribution license which permits unrestricted use, distribution, and reproduction in any medium, provided the original work is properly cited. 